International Journal of Modern Physics A

Vol. 29, No. 28 (2014) 1446005 (15 pages)

(C) World Scientific Publishing Company

DOI: 10.1142/S0217751X14460051

\title{
Study of Double Pomeron Exchange with the SFM detector at the CERN ISR
}

\author{
H. G. Fischer \\ CERN, Geneva, Switzerland \\ hans.gerhard.fischer@cern.ch \\ W. Geist \\ geistwalter@web.de \\ M. Makariev \\ Institute for Nuclear Research and Nuclear Energy, \\ Bulgarian Academy of Sciences, Sofia, Bulgaria \\ martin.makariev@cern.ch
}

Received 28 May 2014

Accepted 30 July 2014

Published 10 November 2014

\begin{abstract}
Selected results from the study of Double Pomeron Exchange with the Split Field Magnet detector at the CERN Intersecting Storage Rings are presented. This concerns various general features of central pionic systems as well as the spectroscopy in the $\pi^{+} \pi^{-}$ channel, emphasizing the production properties of the $f_{2}(1270)$ meson. This summary refers to a series of publications over a time span of 17 years based on the work of the $\mathrm{CCHK}^{1,2}$ and $\mathrm{ABCDHW}{ }^{3-7}$ collaborations at the CERN ISR.
\end{abstract}

Keywords: Pomeron; pion.

PACS number: 13.85.Hd

\section{The Split Field Magnet Detector}

The Split Field Magnet (SFM) spectrometer belongs to the first generation of detector facilities equipped exclusively with Multiwire Proportional Chambers. It was conceived for use at the CERN Intersecting Storage Rings (ISR) and was positioned symmetrically over one of the interaction regions. A sketch of the experimental arrangement is shown in Fig. 1(a) as a top view. Figure 1(b) presents a vertically extended side view of the two forward spectrometers indicating the small angle region used for the detection of fast-forward protons. 

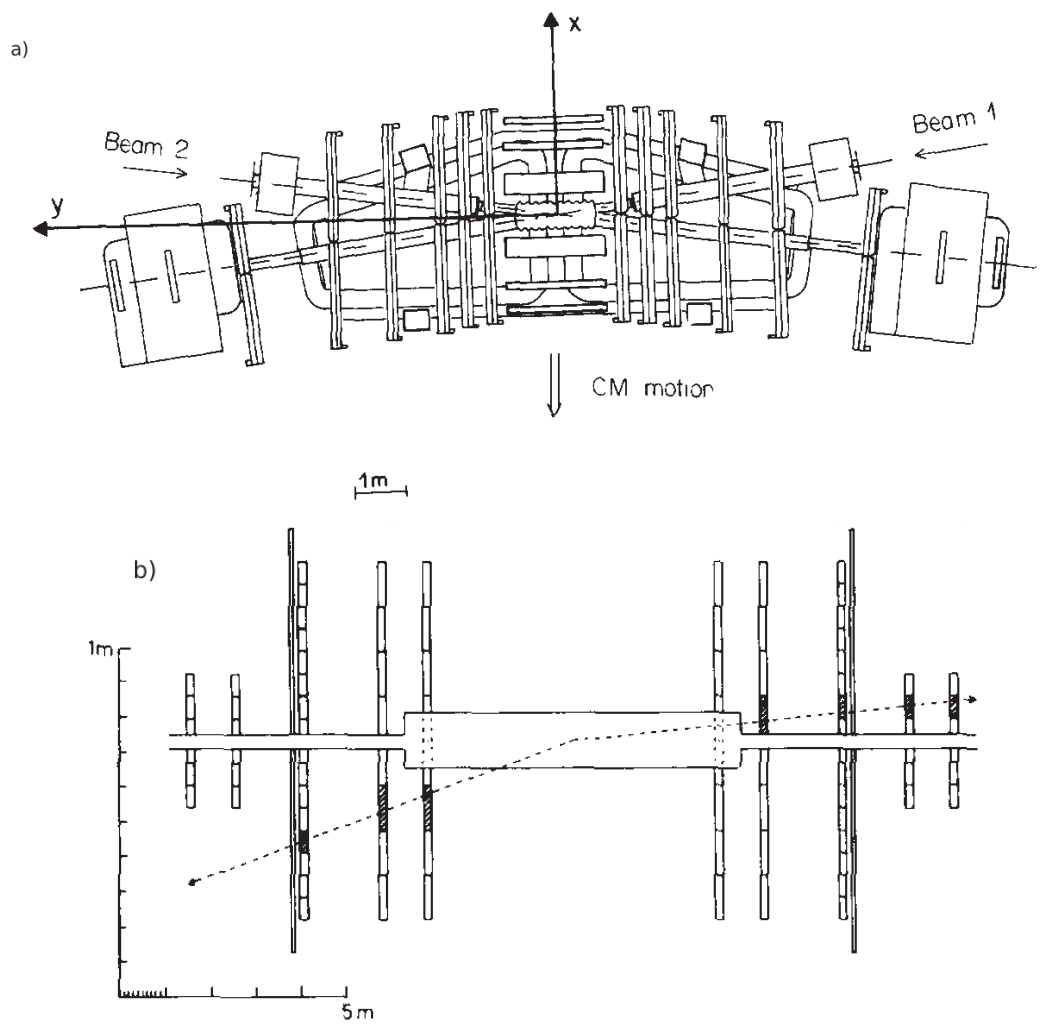

Fig. 1. SFM layout: (a) top view and (b) vertical view.

The detector was equipped with 28 two-plane MWPC's and eight 3-10 plane chambers in the central area with a total surface of $180 \mathrm{~m}^{2}$ and about $60 \mathrm{k}$ electronics channels. The magnet system consisted of two main vertical, opposite fields of 1 Tesla and four compensator magnets restoring the closed orbit of the circulating beams. The acceptance covered more than $95 \%$ of the total solid angle and allowed in particular the efficient rejection of tracks above unit rapidity.

The minimum polar angle of $11 \mathrm{mrad}$ accepted at beam momentum offered a range of squared momentum transfer from -0.03 to $-0.1(\mathrm{GeV} / c)^{2}$ over the range of ISR cms energies, $\sqrt{s}$, from 31 to $62 \mathrm{GeV}$.

\section{Cuts}

The Double Pomeron Exchange (DPE) interaction may be characterized by the production of two fast protons and the existence of a central hadronic system in the reaction $p p \rightarrow p_{1}+(X)+p_{2}$. If the forward protons are selected at Feynman $x_{F}$ above $x_{F 1}$ and $x_{F 2}$ respectively, the effective mass of the central system is given by $M^{2}=s\left(1-x_{F 1}\right)\left(1-x_{F 2}\right)$. For a selection of protons above $x_{F}=0.9$ this allows for a mass range from 3 to $6 \mathrm{GeV}$ at the ISR. The main experimental background 

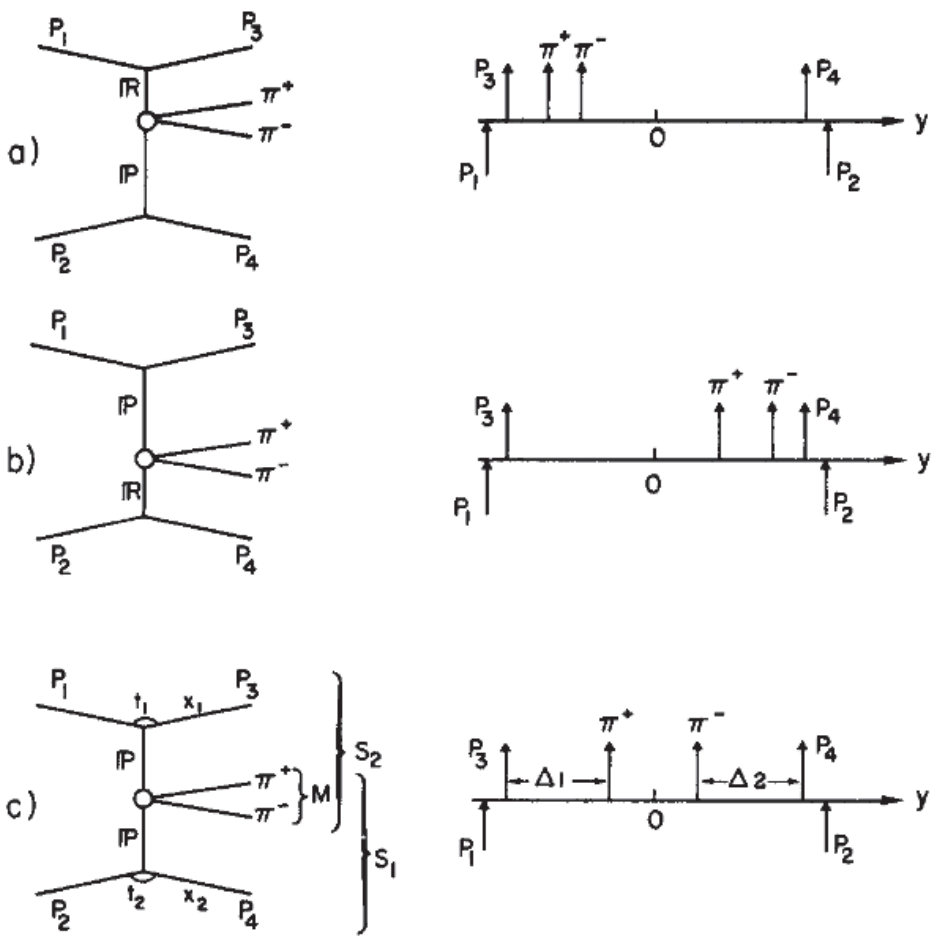

Fig. 2. (a, b) Diffraction dissociation diagrams and (c) DPE for $p p \rightarrow p p \pi^{+} \pi^{-}$.

to the DPE process is caused by single diffraction (SD) on one of the outgoing protons, see Fig. 2 for a central $\pi^{+} \pi^{-}$system.

It is therefore customary to impose rapidity gaps $\left(\Delta_{1}\right.$ and $\Delta_{2}$ in Fig. 2$)$ between the forward protons and the centrally produced secondaries. In order to suppress events with undetected neutral particles a kinematical four-constraint fit was imposed with a $\chi^{2}$ probability above $5 \%$. A resulting rapidity distribution of particles in the above configuration with rapidity gaps $\Delta_{1}=\Delta_{2}$ of two units is shown in Fig. $3 .^{3}$

The ISR energy range is well suited to such selection criteria as at lower energies the clean separation from SD events becomes impossible due to the smaller overall rapidity range, and with increasing energies a clean veto for the intermediate $y$ range is more difficult to establish.

\section{Factorization}

A powerful check of the dominance of the DPE process is the fact that the momentum transfers from the incident protons to the central hadronic system should be uncorrelated. This is shown in Fig. 4 for the scatter plot, in exponential scale, of the momentum transfers $-t_{1}$ against $-t_{2}$. 


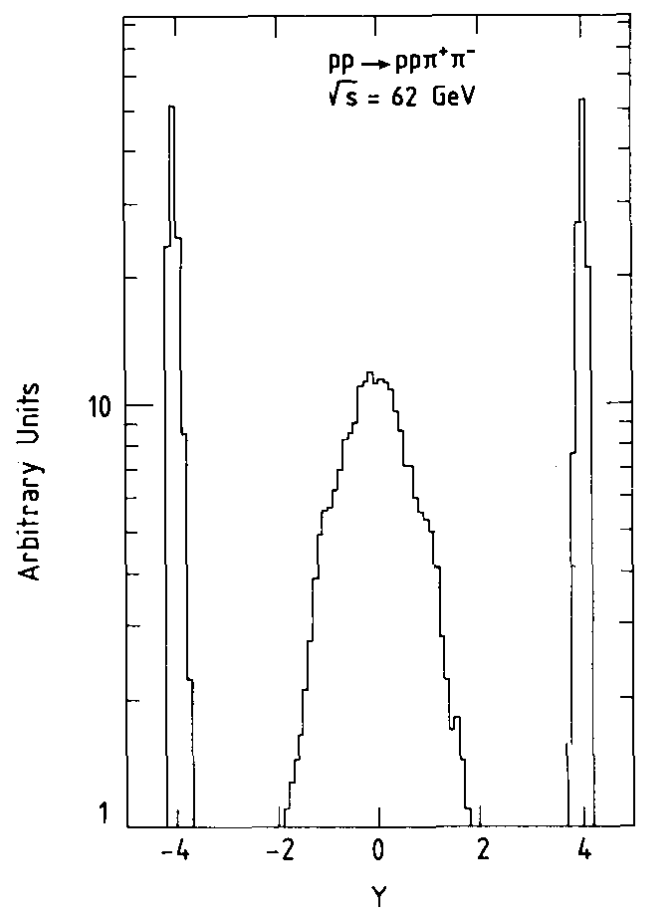

Fig. 3. Rapidity distribution of particles from a 4 -C fit to the reaction $p p \rightarrow p p \pi^{+} \pi^{-}$at $\sqrt{s}=$ $62 \mathrm{GeV}$.

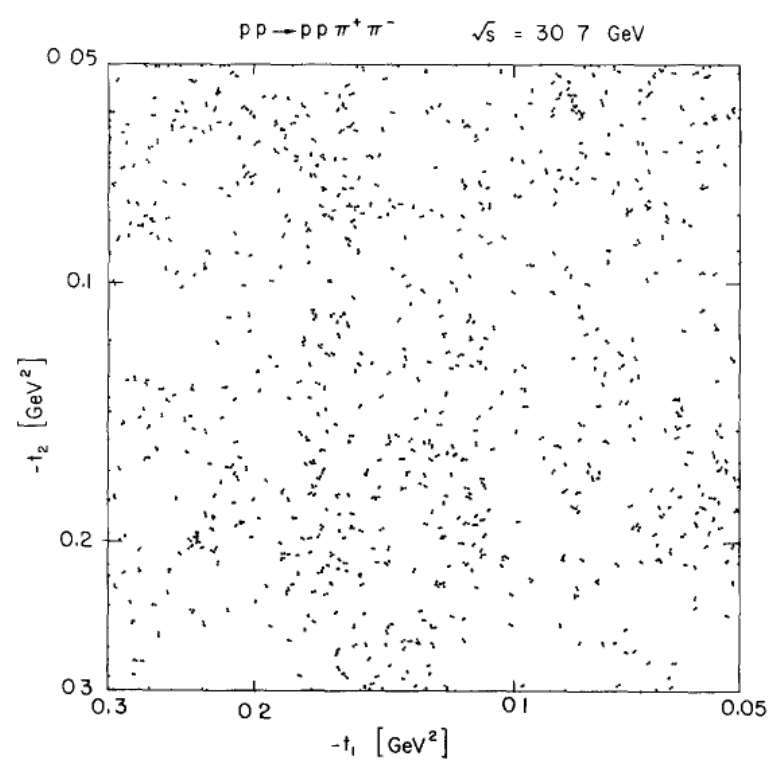

Fig. 4. Scatter diagram of $t_{1}$ versus $t_{2}$ in a double exponential scale. 

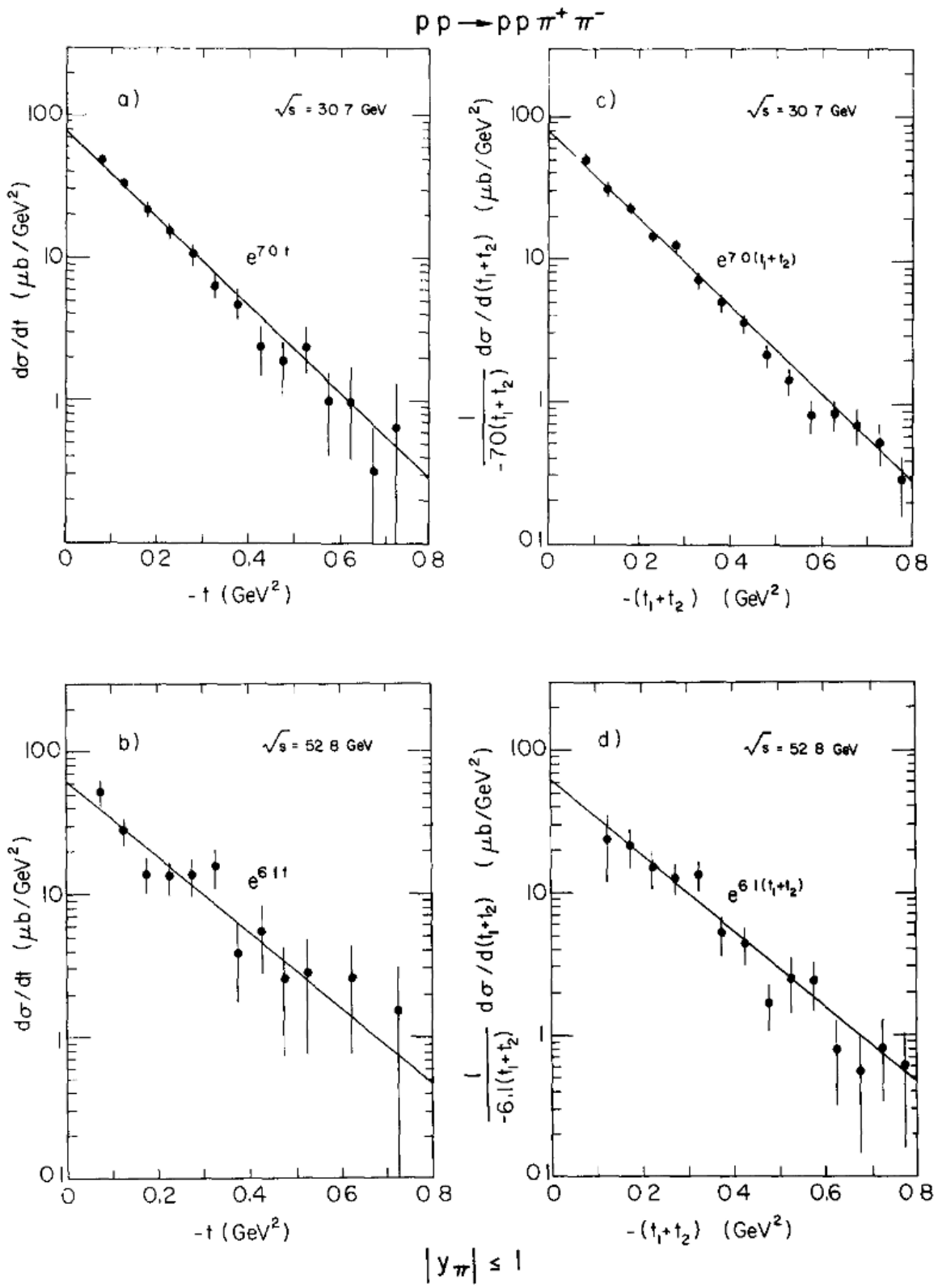

Fig. 5. (a, b) Distribution of $-t$ for $\sqrt{s}$ of 31.7 and $52.8 \mathrm{GeV}$. (c, d) Distribution of the sum of the momentum transfers.

The distributions of $t_{1}$ and $t_{2}$ as well as of their sum $-\left(t_{1}+t_{2}\right)$ should be identical if the latter cross section is given as $1 /-\left(t_{1}+t_{2}\right) * d \sigma / d\left(t_{1}+t_{2}\right)$. As obvious from Fig. 5 for two ISR energies all distributions show compatible slopes. 


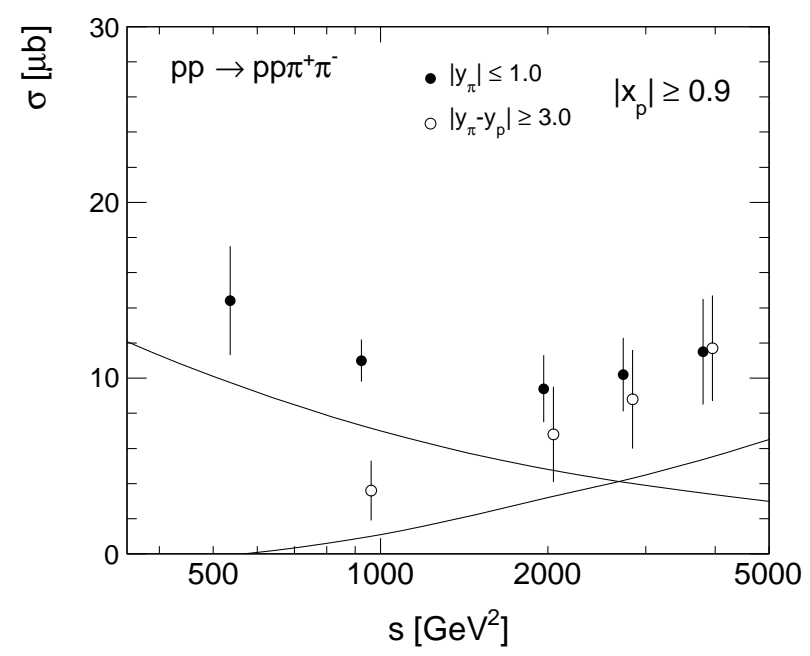

Fig. 6. Cross section of the channel $p p \rightarrow p p \pi^{+} \pi^{-}$as a function of $s$ for two different rapidity cuts for the central pion system. The full lines are predictions from Ref. 8 for the two chosen rapidity windows.

The slopes $B$ of the momentum transfer distributions are exponential over the range $0.05<-t<0.8$ with values in the range from 6 to $7(\mathrm{GeV} / c)^{-2}$ which corresponds to the expectation that the DPE slope should be about $1 / 2$ the slope of elastic scattering.

\section{Cross Section for Events of the Type $p p \rightarrow p p \pi^{+} \pi^{-}$}

The cross section of the central two-pion sample, which has been subjected to a four-constraint fit, is given in Fig. 6 as a function of $s$ for the two different rapidity cuts of $y_{\pi} \leq 1$ at the five standard ISR energies, and $y_{\pi} \leq 1.5$ units at $\sqrt{s}=31 \mathrm{GeV}$. These data are also compared to a theoretical prediction ${ }^{8}$ for these two rapidity intervals.

Given the uncertainties in the assumptions of Ref. 8 and the experimental errors the agreement between prediction and data is satisfactory.

\section{Momentum Distributions}

The central, kinematically well-constrained pion sample discussed above offers a unique chance to follow the evolution of hadronic production from a primordial system with given spin and decay characteristics to the inclusive momentum distribution as it is measured in the overall $p+p$ cms.

Subdividing the effective mass distribution of the two-pion system into bins, and plotting the corresponding transverse momentum distributions $p_{T}^{*}$ in the pionpion (i.e. Pomeron) cms, the histograms shown in Fig. 7 are obtained, where $p_{T}^{*}$ is computed with respect to the incident Pomeron direction. 


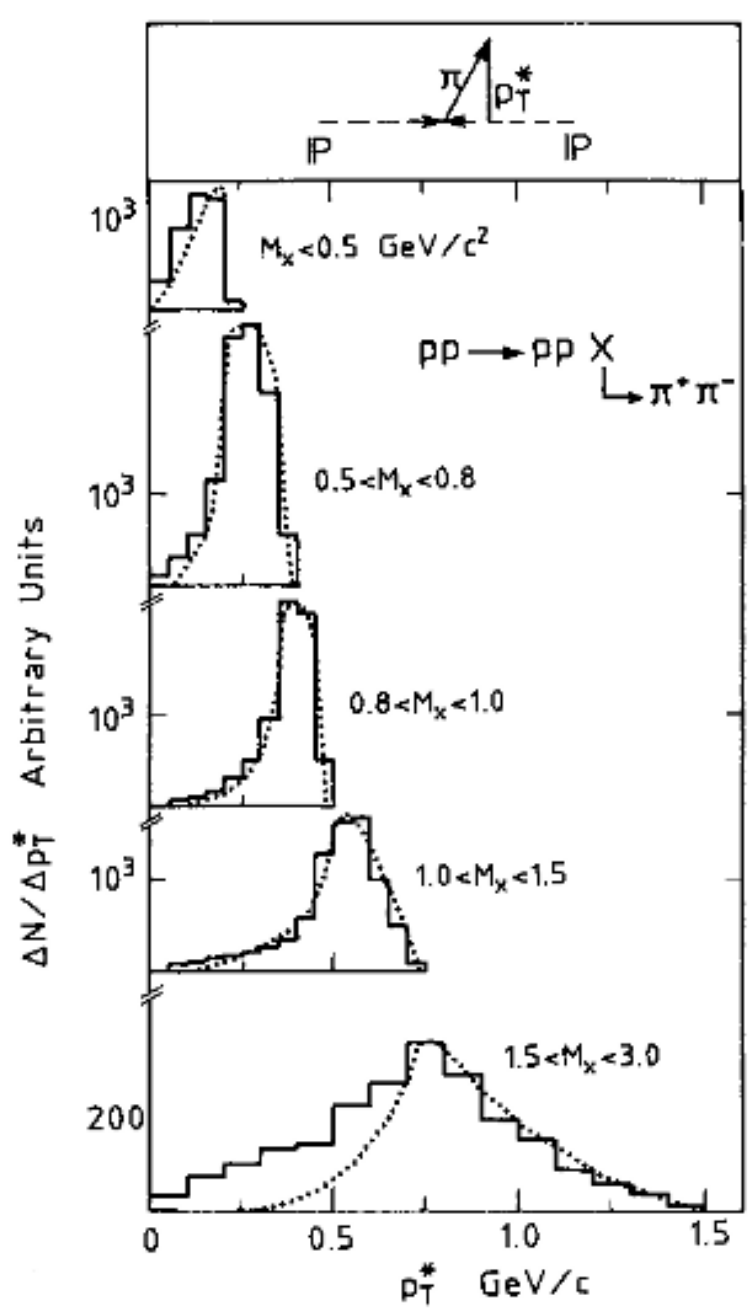

Fig. 7. $p_{T}^{*}$ distribution for central pions in the rest frame of the $\pi^{+} \pi^{-}$system for different slices of $M\left(\pi^{+} \pi^{-}\right) \cdot p_{T}^{*}$ is computed with respect to the incident Pomeron direction. Dashed lines are calculations for the two body decay of the central system.

Superimposed to the experimental histograms are predictions from the phase space decay into two pions of a mass $M_{x}$ at rest, using pure $s$-wave behaviour up to $M_{x}=1 \mathrm{GeV}$ and above $1.5 \mathrm{GeV}$. In the range $1-1.5 \mathrm{GeV}$ a superposition of $s$ and $d$-waves as obtained from a fit to the mass spectrum (Sec. 6) has been used. The $p_{T}^{*}$ distributions are of course sharply limited by the $M_{x}$ bin, showing a pronounced maximum at about $\left\langle M_{x}\right\rangle / 2$.

Transforming these distributions to the $p+p$ rest frame and plotting $1 / p_{T}^{*} \Delta N / \Delta p_{T}$, a different picture emerges, Fig. 8. 


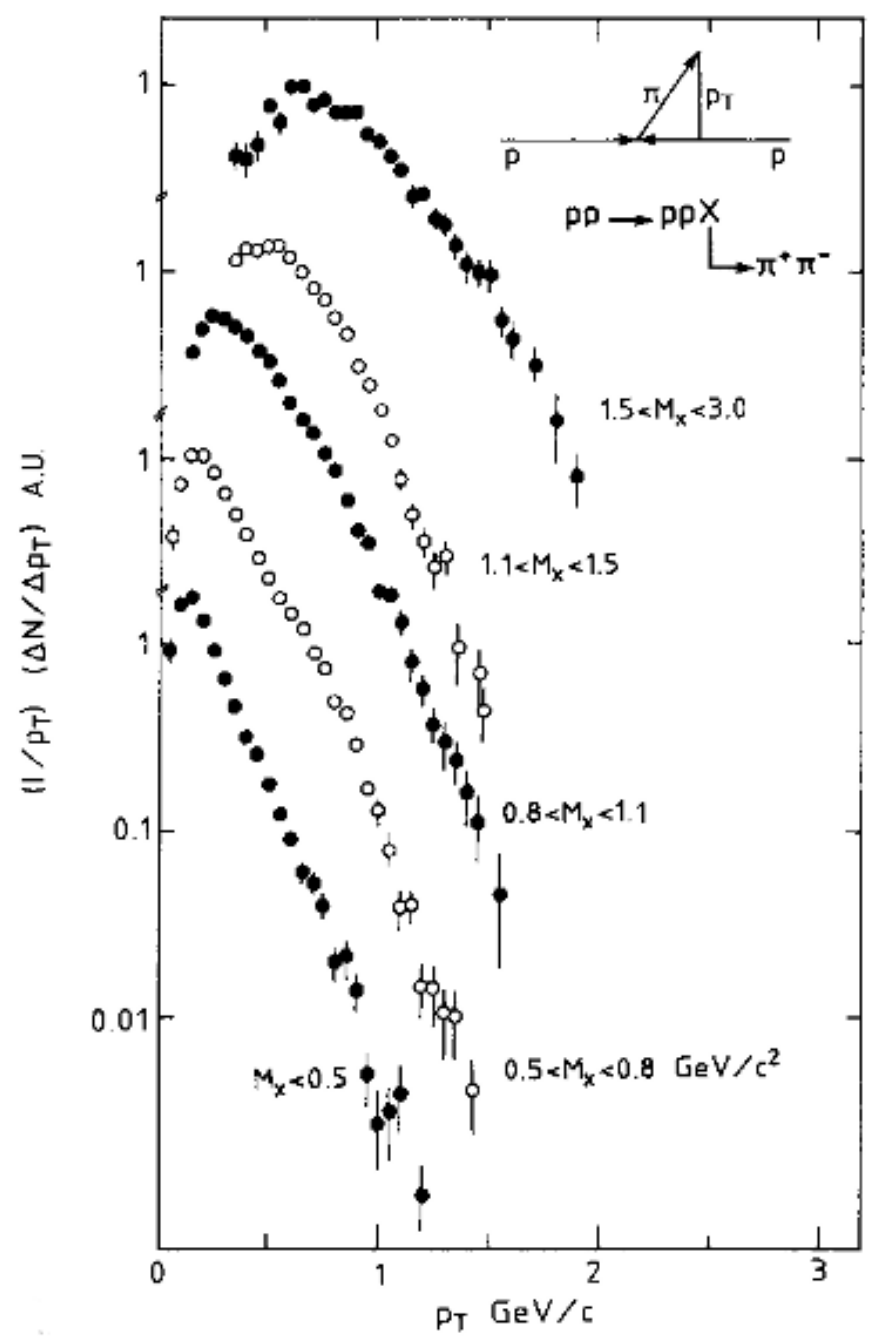

Fig. 8. Pion $p_{T}$ distributions in bins of $M\left(\pi^{+} \pi^{-}\right)$in the proton-proton cms, $p_{T}$ given with respect to the incident proton direction.

As a result of the corresponding Lorentz transformation these $p_{T}$ distributions are extending to $p_{T}$ values above $1 \mathrm{GeV} / c$ already in the lowest $M_{x}$ bin, approaching an exponential shape. Summing up all $M_{x}$ bins up to $M_{x}=3 \mathrm{GeV}$, the distributions shown in Fig. 9 are obtained.

These distributions are exponential above $p_{T} \sim 0.7$ and $0.3 \mathrm{GeV} / c$ for the two and four pion samples, respectively. Their slope at $-5.6(\mathrm{GeV} / c)^{-1}$ corresponds exactly to the slope observed in minimum bias inelastic $p+p$ collisions at the ISR up to $p \sim 1.3 \mathrm{GeV} / c$ as shown in Fig. 10 . 

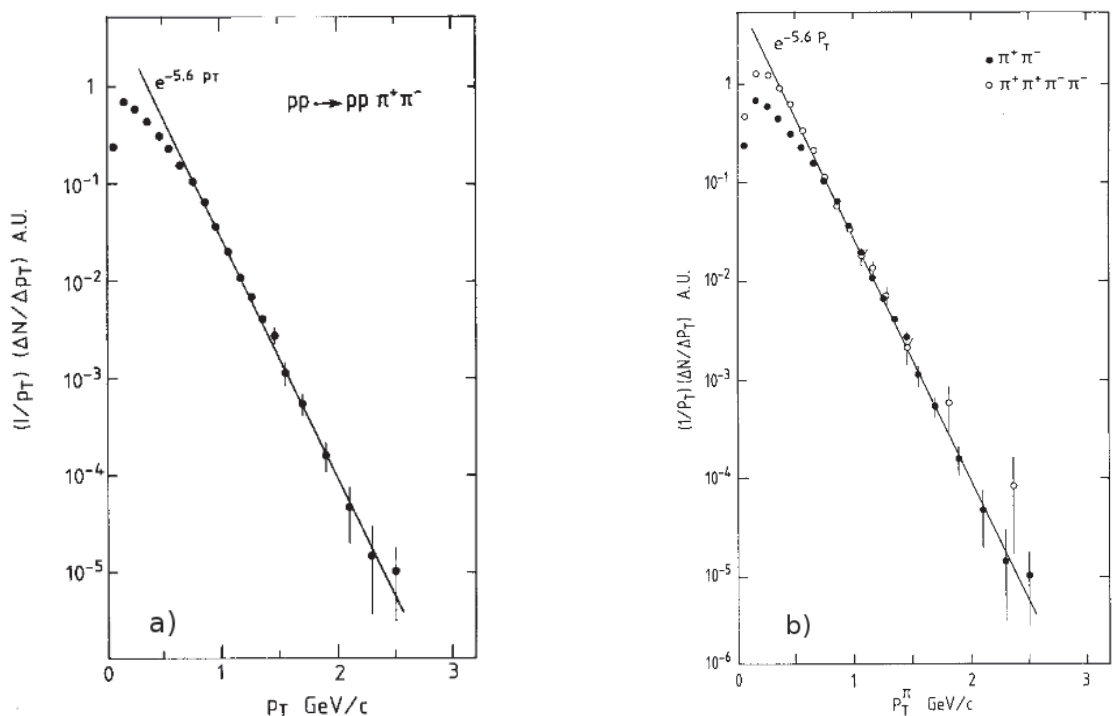

Fig. 9. (a) $p_{T}$ distribution for pions centrally produced in the reaction $p p \rightarrow p p \pi^{+} \pi^{-}$in the $p p$ cms. (b) The same distribution plotting in addition the four pion channel $p p \rightarrow p p \pi^{+} \pi^{-} \pi^{+} \pi^{-}$. The distributions are exponential above $p_{T} \sim 0.7$ and $0.3 \mathrm{GeV} / c$ for Figs. 9(a) and 9(b), respectively, with a common slope of $-5.6(\mathrm{GeV} / c)^{-1}$.

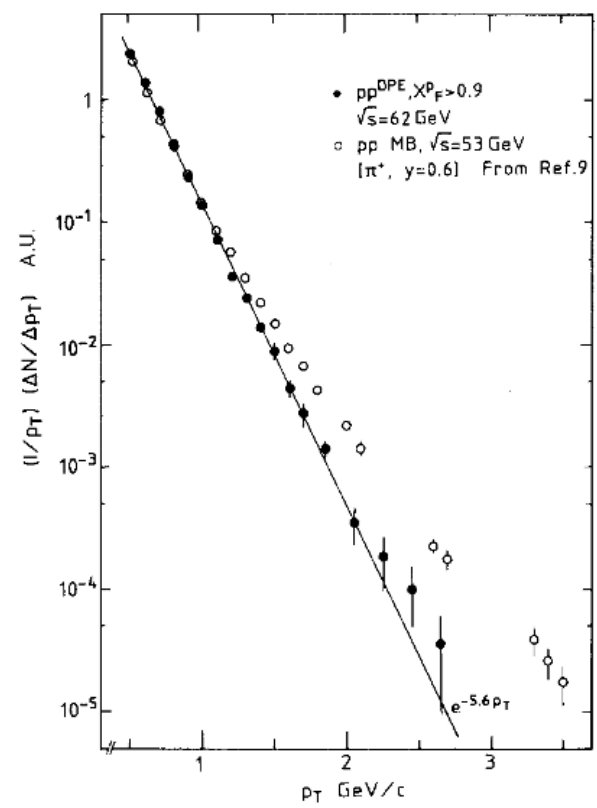

Fig. 10. Inclusive $p_{T}$ distributions for centrally produced pions in events of the type $p p \rightarrow p p+2 \pi$ and $p p+4 \pi$ at $\sqrt{s}=62 \mathrm{GeV}$ with final state protons at $x_{F}>0.9$ (full dots). The data are compared with the inclusive $p_{T}$ distribution for minimum bias inelastic $p+p$ interactions (open dots). The distributions are normalized to each other at $p_{T}=1 \mathrm{GeV} / c$. 
The deviation of the minimum bias data from exponential slope above $p_{T} \sim$ $1.3 \mathrm{GeV} / c$ has been ascribed to hard parton scattering. In view of the fact that the available effective mass due to the trigger condition for the fast protons is only $6 \mathrm{GeV}$ as compared to much larger values for the minimum bias data, this effect may equally well be ascribable to the production of higher mass resonances and their subsequent decay into pions. In fact it has been shown that the shape of the transverse momentum distributions at SPS energies is well reproduced up to $3 \mathrm{GeV} / c$ by the decay of heavy resonances. ${ }^{9}$

A further interesting result is obtained when looking at the average longitudinal and transverse momentum of the proton momentum transfer (i.e. the Pomeron momentum) in the $p+p$ cms as a function of the central two-pion mass $M\left(\pi^{+} \pi^{-}\right)$, Fig. 11.

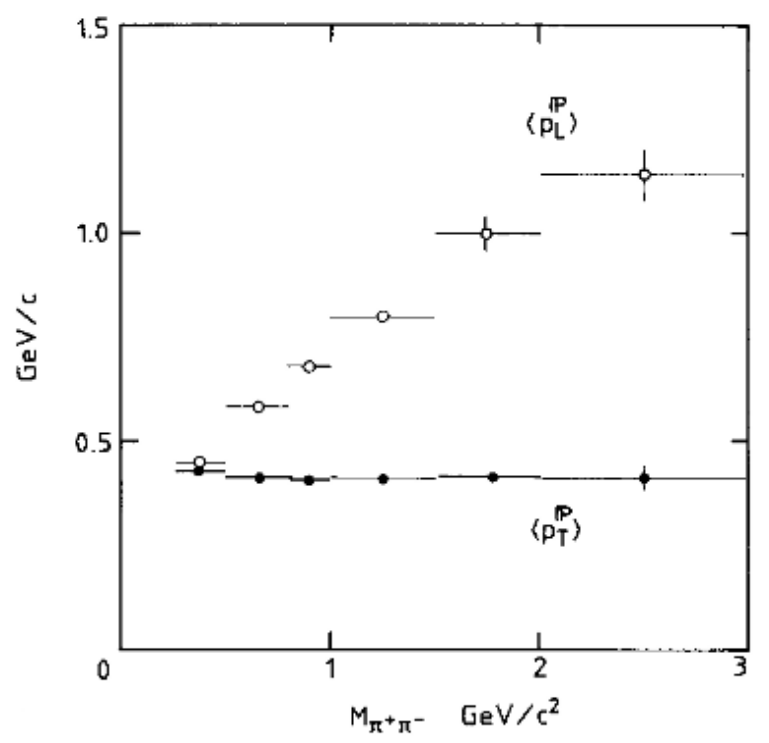

Fig. 11. Average transverse and longitudinal components of the proton momentum transfer (Pomeron momentum) in the $p p$ cms as a function of the mass of the central $\pi^{+} \pi^{-}$system.

This result shows that the average transverse momentum of the Pomeron is independent of $M\left(\pi^{+} \pi^{-}\right)$at a value of about $0.4 \mathrm{GeV} / c$ whereas the average longitudinal momentum increases linearly with $M\left(\pi^{+} \pi^{-}\right)$up to values above $1 \mathrm{GeV} / c$, indicating the longitudinal nature of the Pomeron emission.

\section{Spectroscopy}

The spectroscopy of the central two-pion system in DPE reactions offers further insight into the exchange mechanism, in particular concerning the fact that only 
states with the quantum numbers $J^{\mathrm{PC}}=N^{++}$with $N$ even may be produced. This allows, at ISR energies, essentially the study of $s$ and $d$-wave contributions and the verification of the absence of $J=1$ states which are abundantly present in the background of single and double diffraction. ${ }^{10}$ In addition, the opening of the kaon pair threshold should affect the measured mass spectrum in the region around $1 \mathrm{GeV}$. As Pomeron-Pomeron scattering is assumed to be dominated by gluons, the spectroscopy of the corresponding central hadronic system has also been considered as a possibility to study bound states of gluons (glue-balls).

The invariant $\pi^{+} \pi^{-}$mass distribution obtained at $\sqrt{s}=62 \mathrm{GeV}$ is shown in Fig. 12 for the trigger conditions discussed in Sec. 2, Fig. 3 with forward protons at $x_{F}>0.9\left(t \geq 0.08 \mathrm{GeV}^{2}\right)$ and two central pions limited to the region $y<1.5 .^{3}$ The experimental sample was subjected to a kinematic four-constraint fit.

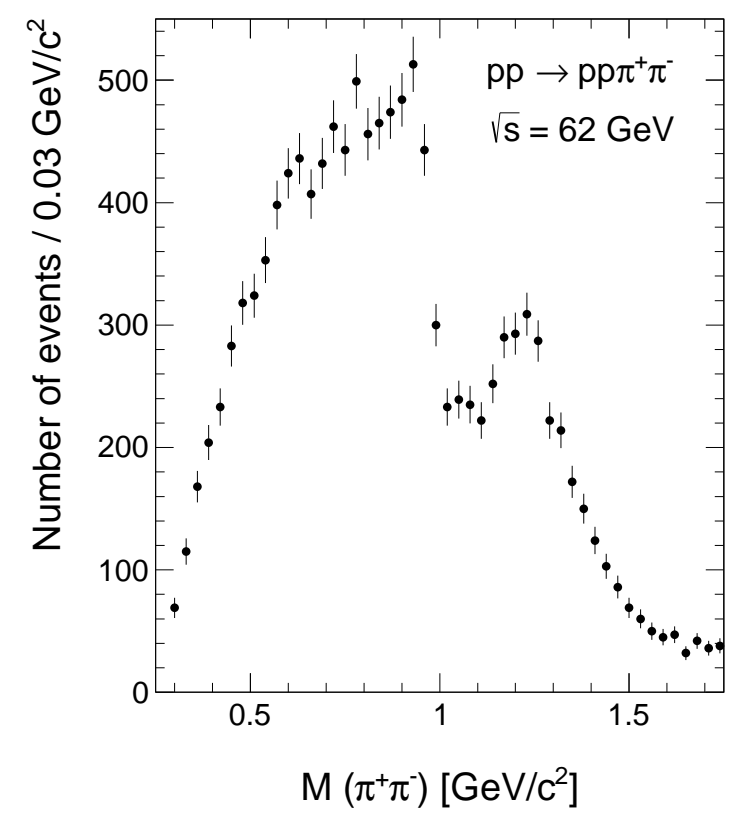

Fig. 12. Invariant mass distribution of the central $\pi^{+} \pi^{-}$system for events with fast protons at $x_{F}>0.9$ and central pions with $y<1.5$ units. $^{3}$

The mass spectrum shows a broad structure rising from the two-pion threshold up to about $0.96 \mathrm{GeV}$. This rise is followed by a sharp drop at the two-kaon threshold and a resonant structure above $1.1 \mathrm{GeV}$. The absence of any indication of the presence of the $\rho^{0}$ meson indicates that the event selection indeed suppresses the diffractive background very effectively. The eventual appearance of the allowed $f_{0}$ state at $0.98 \mathrm{GeV}$ is evidently strongly affected by the onset of kaon pair production in this mass region and a coupled-channel analysis of the $\pi^{+} \pi^{-}$and $\mathrm{K}^{+}+\mathrm{K}^{-}$mass spectra is needed to clearly describe the structure seen in the data. ${ }^{11}$ 


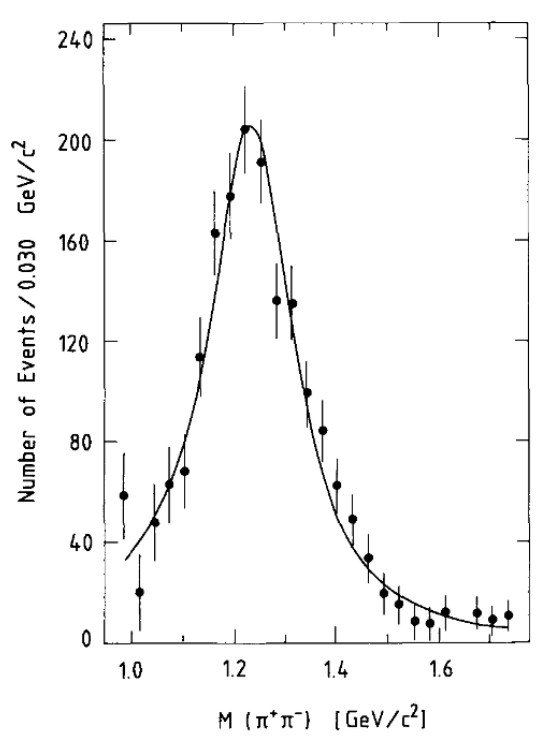

Fig. 13. Invariant mass distribution for the central $\pi^{+} \pi^{-}$system after the subtraction of a nonresonant background.

In order to interpret the resonant structure above $1.1 \mathrm{GeV}$ a steeply falling background has to be taken into account. Describing this background by the form $A * M\left(\pi^{+} \pi^{-}\right)^{-\beta}$ with $\beta=3.9 \pm 0.3$ the mass spectrum of Fig. 13 is obtained.

A fit to this mass spectrum results in a resonance mass of $1.279 \pm 0.010 \mathrm{GeV} / c^{2}$ with a width of $0.220 \pm 0.030 \mathrm{GeV} / c^{2}$ which reduces to $0.196 \pm 0.034 / c^{2}$ taking into account the calculated mass resolution of $0.050 \mathrm{GeV}$. These parameters are in good agreement with the mass and width of the $f_{2}(1270)$ tensor meson. A similar analysis using the SFM detector has been performed by the CHOV collaboration ${ }^{12}$ at $\sqrt{s}=53$ and $63 \mathrm{GeV}$, with corresponding cuts in proton $x_{F}$ and pion rapidity and a range of momentum transfer reaching down to $t=0.033 \mathrm{GeV}^{2}$ enhancing the mass spectrum in the threshold region.

The two-pion mass spectrum has been decomposed into $s$ and $d$-wave components by parametrizing the polar angle distribution in terms of the corresponding density matrix elements. ${ }^{6}$ The resulting $s$ and $d$-wave cross sections are shown in Fig. 14.

The prevalence of the $s$-wave in the low mass region and the preponderant presence of the $d$-wave in the $f_{2}(1270)$ region are clearly evident.

\section{Breakdown of Factorization at the $f_{2}(1270)$ Resonance}

The factorization at the Pomeron vertices predicts the absence of correlation between the two outgoing protons, except for that from kinematics and the trigger acceptance. Determining the azimuthal angle $\Delta \Phi$ between the final state protons 

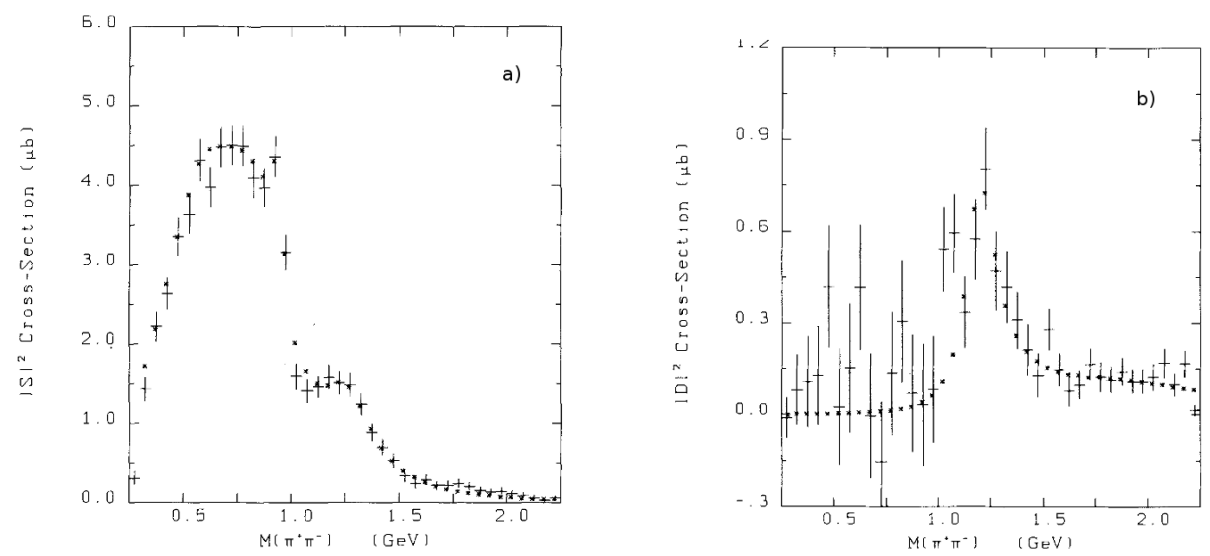

Fig. 14. Cross sections for (a) $s$-wave and (b) $d$-wave contributions to the mass spectrum as a function of the two-pion mass.

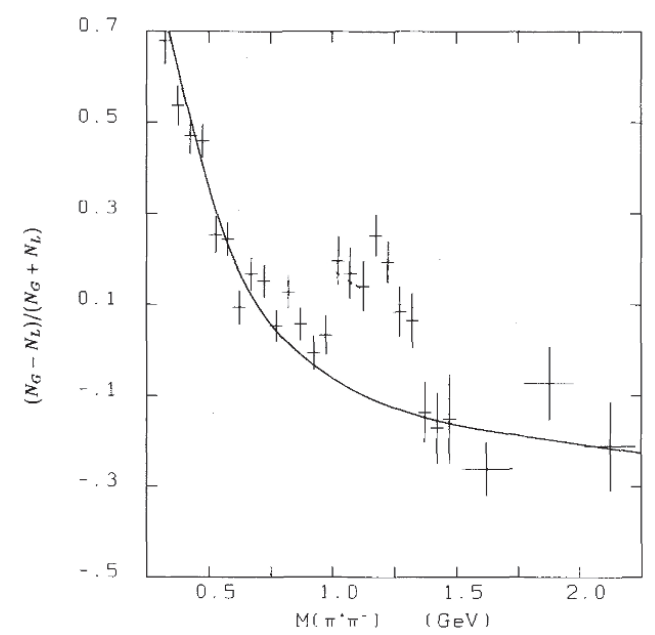

Fig. 15. Azimuthal asymmetry $R=\left(N_{G}-N_{L}\right) /\left(N_{G}+N_{L}\right)$ as a function of $M\left(\pi^{+} \pi^{-}\right)$for uncorrected data. The full line shows the prediction of a DPE model.

in the $p p \mathrm{cms}$, one may define an asymmetry ratio $R$ as follows:

$$
R=\frac{N_{G}-N_{L}}{N_{G}+N_{L}}
$$

as a function of $M\left(\pi^{+} \pi^{-}\right)$where $N_{G}\left(N_{L}\right)$ is the number of events with $\Delta \Phi$ greater (respectively, less) than 90 degrees. This ratio is shown in Fig. 15 for the uncorrected data and for the prediction of a DPE model including the experimental acceptance. ${ }^{6}$

The data show a clear deviation from factorization in the region of the $f_{2}(1270)$. This effect was further analysed performing a partial wave analysis separately for the samples $N_{G}$ and $N_{L}$, including acceptance corrections. The result is shown 

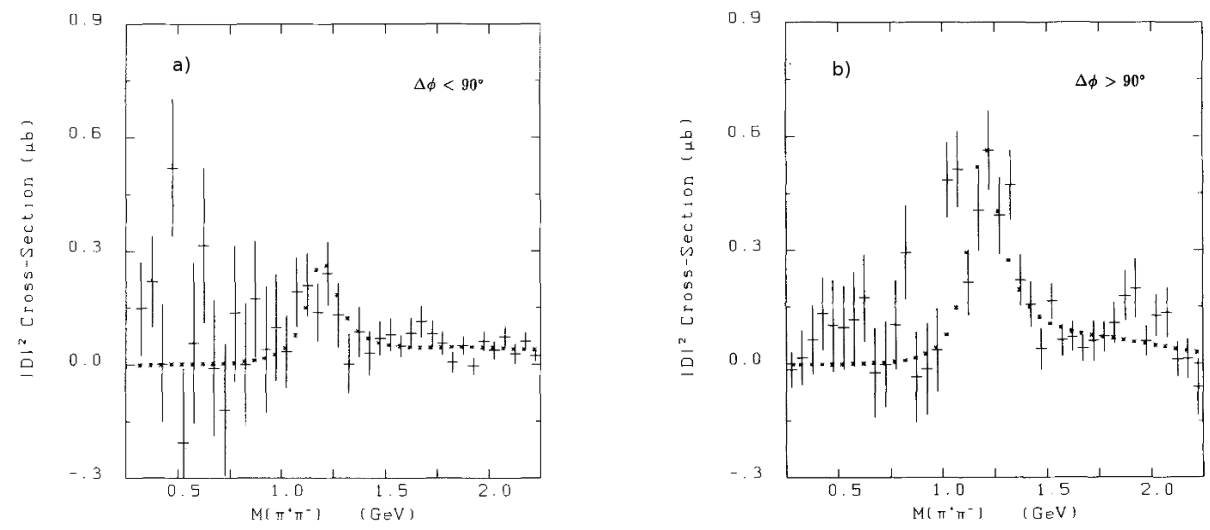

Fig. 16. Acceptance corrected cross section for the $d$-wave as a function of $M\left(\pi^{+} \pi^{-}\right)$for (a) $\Delta \Phi<90$ degrees and (b) $\Delta \Phi>90$ degrees.

for the $d$-wave cross sections in Fig. 16 for the selections $\Delta \Phi<90$ and $\Delta \Phi>90$ degrees.

The cross section for $\Delta \Phi>90$ degrees exceeds the one for $\Delta \Phi<90$ degrees by more than a factor of two whereas for the $s$-wave (not shown) there is only a difference of $10-15 \%$. A similar effect has been observed by the WA91 collaboration ${ }^{13}$ at the CERN SPS at the lower energy of $\sqrt{s}=28 \mathrm{GeV}$.

The interpretation of this phenomenon is still open to speculation. It has been argued that the $f_{2}(1270)$ might have a glue-ball component favouring multiple gluon exchange between the two outgoing protons including a direct exchange term breaking factorization.

\section{Conclusion}

DPE has been studied at the CERN ISR using the Split Field Magnet detector. This instrument offered an open geometry covering most of the production phase space thus allowing for an efficient selection of events with two fast-forward protons and a well-defined central pionic system. The study of the corresponding event samples covered both the dynamical aspects of DPE including some characteristics of the Pomeron, and the spectroscopy of the central two-pion system revealing an unusual production mechanism for the $f_{2}(1270)$ meson.

\section{References}

1. M. Della Negra et al., Phys. Lett. B 65, 394 (1976).

2. D. Drijard et al., Nucl. Phys. B 143, 61 (1978).

3. A. Breakstone et al., Z. Phys. C 31, 185 (1986).

4. A. Breakstone et al., Z. Phys. C 40, 41 (1988).

5. A. Breakstone et al., Z. Phys. C 42, 387 (1989).

6. A. Breakstone et al., Z. Phys. C 48, 569 (1990).

7. A. Breakstone et al., Z. Phys. C 58, 251 (1993). 
8. D. M. Chew and G. F. Chew, Phys. Lett. B 53, 191 (1974); D. M. Chew, Phys. Lett. B 65, 367 (1976).

9. http://cern.ch/spshadrons, document S8.

10. C. Conta et al., Nucl. Phys. B 175, 97 (1980).

11. S. M. Flatte et al., Phys. Lett. B 38, 232 (1972).

12. R. Waldi et al., Z. Phys. C 18, 301 (1983).

13. D. Barberis et al., Phys. Lett. B 388, 853 (1996). 